\title{
Chronotherapy of cancer: epithelial-mesenchymal transition
}

\begin{abstract}
Metastasis is resistant to conventional therapies and the main cause of death by cancer. In this article we discuss a non-autonomous model for the stage of cancer metastasis in which a periodic perturbation is introduced simulating the action of chronotherapy on the immune system and on the flow rate of the epithelial-mesenchymal transition. As a result of the perturbation, regions of less complexity are obtained, which implies less robustness or plasticity. These results allow us to delineate strategies for the chronotherapautic treatment in cancer metastasis.
\end{abstract}

Keywords: chronotherapy, cancer, EMT, complexity
Volume 4 Issue 4 - 2019

\author{
A Guerra,' DJ Rodriguez,' E Silva,' JA \\ Betancourt-Mar, ${ }^{2}$ G Cocho, ${ }^{3}$ R Mansilla, ${ }^{4}$ JM \\ Nieto-Villar' \\ 'Department of Chemical-Physics, A Alzola Group of \\ Thermodynamics of Complex Systems of MV Lomonosov Chair, \\ Faculty of Chemistry, University of Havana, Cuba \\ ${ }^{2}$ Mexican Institute of Complex Systems, Tamaulipas, México \\ ${ }^{3}$ Instituto de Física de la UNAM, México \\ ${ }^{4}$ Centro de Investigaciones Interdisciplinarias en Ciencias y \\ Humanidades, UNAM, México
}

Correspondence: JM Nieto-Villar, Department of ChemicalPhysics, A Alzola Group of Thermodynamics of Complex Systems of MV Lomonosov Chair, Faculty of Chemistry, University of Havana, Cuba, Email nieto@fq.uh.cu

Received: May 07, 2019 | Published: July 26, 2019
Abbreviations: EMT, epithelial-mesenchymal transition; MET, mesenchymal-epithelial transition; ODE, ordinary differential equation

\section{Introduction}

Cancer is a complex system that self-organizes far from thermodynamic equilibrium, exhibiting high plasticity and adaptability. ${ }^{1}$ It goes through three fundamental growth stages: avascular, vascular and metastasis, ${ }^{2}$ of all of them metastasis is the deadliest. ${ }^{3}$ In this stage the tumor invades nearby or distant organs.

The epithelial-mesenchymal transition (EMT) plays a fundamental role in embryonic development and metastasis. ${ }^{4}$ The metastasis of cancer is not only the most fatal stage but also the most complex one. ${ }^{5}$ During this process cell-cell interaction and cell-membrane basal interaction is lost, this implies losing the polarity and the typical forms of the epithelial phenotypes, which occurs through genotypic changes. ${ }^{6}$ The cells acquire then a Mesenchymal phenotype characterized by a high capacity for invasion and resistance to apoptosis. ${ }^{7}$

The reverse process of EMT is the mesenchymal-epithelial (MET) transition. Although this process occurs during normal development and reprogramming of stem cells, it is also related to the metastasis of cancer and the state of dormancy. ${ }^{8-10}$

An alternative to cancer treatments is chronotherapy, which consists in the application of a therapy taking into account the biological rhythms. This has been applied in cancer patients with positive results. ${ }^{11,12}$ Generic and cancer models have been developed using systems of differential equations, which include the effect of chronotherapy. ${ }^{13-16}$ It has been proved that external perturbations in some cases can be coupled with biological rhythms causing a decrease in temporal complexity. ${ }^{16}$ However, as far as we know there is no model that allows to relate the frequency and dose of an immunotherapy treatment in the stage of metastasis during the Epithelial-Mesenchymal transition. Therefore, the objective of our work is to propose a non-autonomous model of ordinary differential equations, based on a model previously developed by our research group. ${ }^{5}$

\section{The methodology of work}

Mathematical modeling of tumor growth makes it possible to describe their more regularities and it provides us with information for the application of more effective therapies, the development of drugs and taking clinical decisions against cancer. ${ }^{17,18}$ In this case, the modeling of two processes was carried out: immunotherapy and rate of EMT flow.

The immunotherapy, also called biologic therapy, is a type of cancer treatment that stimulates the organism's natural defenses in order to fight it. ${ }^{19}$ Using substances produced by the organism or manufactured in a laboratory to improve or restore the function of the immune system..$^{20}$ The immunotherapy can work in different ways: Stop or delay the growth of cancer cells or prevent spreading cancer to other organs. Monoclonal antibodies are a specific type of drug, which are manufactured in a laboratory. They can be used in various ways. For example, monoclonal antibodies can be used in a targeted therapy to block an abnormal protein in a cancer cell. ${ }^{21}$

It is known that the variation of the EMT alter drastically the behaviour of the metastatic tumour population, ${ }^{22}$ which suggests that EMT is a good checkpoint to treat cancer in this stage. For the modelling of both treatments, simulating a chronotherapeutic regime, periodic perturbations are introduced in a cancer model (Figure 1). 


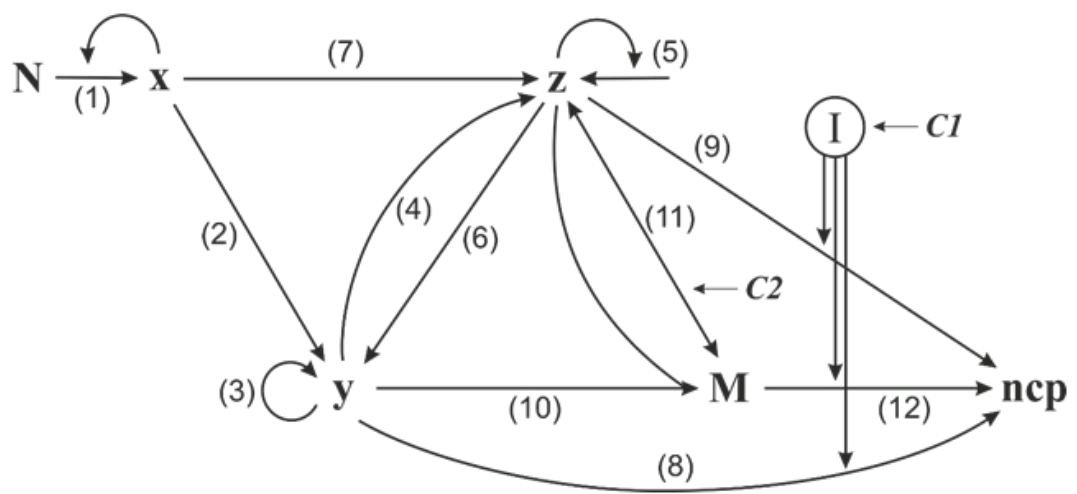

Figure I The network model for chronotherapy of the EMT.

In the model $\mathrm{N}$ represents the population of normal cells exposed to the tumour proliferation, $\mathrm{H}$ is the population of host cells in the surrounding environment; ${ }^{22} \mathrm{I}$ is the cell population of the immune system ( $\mathrm{T}$ lymphocytes and natural killer cells), and we use $\mathrm{I}=0.4{ }^{23}$ $\mathrm{N}$ and $\mathrm{H}$ are considered constants because the number of these cell populations is greater that the tumor cells and for practical purposes their number does not vary.

The variables $\mathrm{x} ; \mathrm{y} ; \mathrm{z}$ represents the epithelial cell population in the a vascular, vascular and metastasis stages respectively, $M$ is the mesenchymal cell population. The parameter k11 of EMT is a flow rate and its value is $0.1 .^{24}$

The magnitudes $\mathrm{C} 1$ and $\mathrm{C} 2$ represent the perturbation to each of the control parameters. The control parameters determine the quality the dynamic of the system. This perturbation represents the periodic action of a treatment and it is a sinusoidal function.

Finally, ncp represents a non-carcinogenic product due to the interaction with the immune system. These steps shown in the model are related to processes that occur at the cellular level, mitosis, apoptosis and the form of tumor invasion through the EMT. The value of each of the constants was taken from our previous work. ${ }^{5}$

\section{Results and discussion}

To model the immuno-chronotherapeutics treatment, the sinusoidal function $\mathrm{C} 1$ is added as shown in figure 1. To solve the ODE system (1), the software COPASI (version 4.6.32) was used. However, the numerical integration was made through the implementation of the Gear algorithm for stiff equations, in Fortran with double precision and tolerance of $10-8 .{ }^{24}$ For the characterization of the dynamics, the power spectrum and the value of the exponents of Lyapunov were used.

The term A corresponds to the amplitude of the perturbation; in medical terms it would represent the dose and $\mathrm{f}$ is the autonomous frequency. The $\mathrm{n}$ represents a frequencies relation between the autonomous frequency and the perturbation frequency. Finally, $\mathrm{t}$ represents the time. The non-autonomous ordinary differential equations system is shown in (equation 1).

$$
\begin{gathered}
\frac{d x}{d y}=x(2 N-x)-H x z \\
\frac{d y}{d t}=y(4-0.14 y)+0.5 x^{2}-\left(I_{0}+A \sin ^{2}(\pi f n t)\right) y-y z(0.1+0.5 H)+0.001 z^{2} \\
\frac{d z}{d t}=-\left(I_{0}+A \sin ^{2}(\pi f n t)\right) z+0.07 y^{2}-y z(0.1+0.5 H)-k_{11}-0.002 z^{2} \\
\frac{d M}{d t}=0.1\left(y z-\left(I_{0}+A \sin ^{2}(\pi f n t)\right) M\right)+k_{11}
\end{gathered}
$$

For Solving the ODE system (1), for the value of $\mathrm{k} 11=0.1$ and $\mathrm{I}=0.4$ is shown in Figure 2.

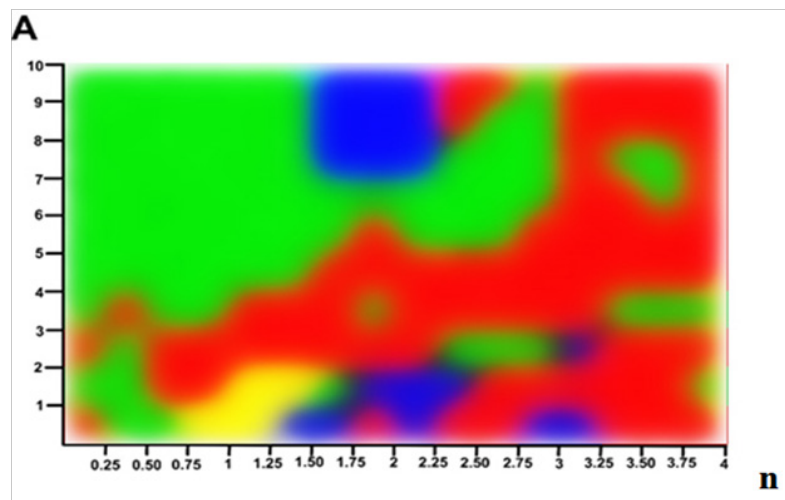

Figure 2 Bifurcation diagram for the ODE system (I) for the value of $\mathrm{k}_{\mathrm{I}}=0 . \mathrm{I}$ and $\mathrm{I}=0.4$. The zones with red color represent the states with chaotic dynamics, the zones in green color represent the quasi-periodic states and the zones in blue represent the limit cycle states. 
The $\mathrm{x}$ axis represents the variation of the frequency values (n) and the $\mathrm{y}$-axis represents the different doses $\mathrm{A}$. The red and green zones are the predominant ones. The second ones represent states with a quasiperiodic dynamic and they do not have a defined oscillation period. They are a possible route to chaos. ${ }^{26}$ The blue regions are the useful ones, because they reduce the complexity of the system to a periodic state.

When the cancer is in the stage of metastasis, it uses the mesenchymal epithelial transition in order to circumvent the body's immune surveillance, as one of its strategies for the proliferation of the primary tumor and a secondary tumor. ${ }^{27}$ It can be observed in the bifurcation diagram (Figure 3) that, only in a small area of high doses and intermediate frequencies, the complexity of the system is reduced, to obtain a better result with this therapy or any other combined. This result corresponds to that reported in literature, where discrete advances have been made in the application of immunotherapy to tumors with advanced stages. ${ }^{28}$

\section{A}

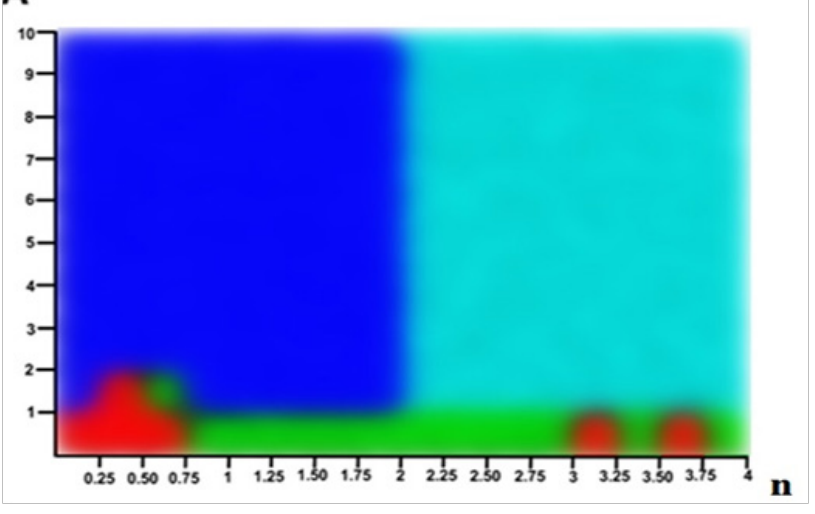

Figure 3 Bifurcation diagram for the ODE system (2) for $\mathrm{k}_{1 \mathrm{I}}=0 . \mathrm{I}$ and $\mathrm{I}=0.4$. The zones with red color represent the chaos, the zones in green color represent the quasi-periodic states and the zones in blue represent the states in the limit cycle.The different tonalities of blue represent the amplitude of the oscillations, the lighter the less one oscillations and so on.

The addition of the perturbative term $\mathrm{C} 2$ to the EMT flow constant is shown below and the same mathematical treatment is performed with the ODE system as shown in equation (2).

$$
\begin{gathered}
\frac{d x}{d y}=x(2 N-x)-H x z \\
\frac{d y}{d t}=y(4-0.14 y)+0.5 x^{2}-I_{0} y-y z(0.1+0.5 H)+0.001 z^{2} \\
\frac{d z}{d t}=-I_{0} z+0.07 y^{2}-y z(0.1+0.5 H)-\left(k_{11}+A \sin ^{2}(\pi f n t)\right)-0.002 z^{2} \\
\frac{d M}{d t}=0.1\left(y z-I_{0} M\right)+\left(k_{11}+\operatorname{Asin}^{2}(\pi f n t)\right)
\end{gathered}
$$

In figure 3 it is shown a bifurcation diagram of the dynamics resulting from the perturbation to the reaction of the EMT (eq. (2)). As can be seen appear a bigger region that exhibit the less complexity comparing with figure 2 .
For values less than $A=1$, states of chaos and quasi-periodic are obtained, which implies zones of more complex dynamics than the limit cycle ones. As observed, treatments aimed at perturbing the EMT at low doses and frequencies can be performed, which would be less invasive and harmful for patients.

Cancer, as a first step to the metastatic cascade, manages to give greater mobility to tumor cells as well as a greater invasive capacity using the EMT. ${ }^{29}$ Thus, it provides cells with a new phenotype with greater resistance to therapies and apoptosis.

Influencing the EMT can achieve better benefits in the treatment of this disease. Some authors have recently suggested seeking therapies that use the transition to mesenchymal cells as a target. ${ }^{30}$ To achieve this, it must be considered the existence of hybrid states during the $\mathrm{EMT}^{31}$ and the current understanding of EMT from mathematical models of regulatory networks and existing experimental systems. ${ }^{32}$ Other studies are aimed at the prognosis and determination of the stage of tumours through markers of EMT. ${ }^{33}$

\section{Conclusions and remarks}

The results obtained allow the optimization of existing therapies, during metastasis. This is useful for patients whose physical conditions (age, sex, conditions, etc.) do not allow the use of conventional therapies such as chemotherapy or high frequency radiotherapy. They can also have a significant impact in terms of toxicity and recovery from the therapy because it is possible to obtain favourable results at low frequencies and amplitudes. In summary, it is found that: The most efficient treatment strategy is the one performed to chronoterapeutically disrupt the mesenchymal epithelial transition, due to less complexity of the system is obtained, which leads to less plasticity and adaptability of the cancer in the stage of metastasis. These results allow us to delineate strategies for the chronotherapeutic treatment in cancer metastasis.

\section{Funding}

None.

\section{Acknowledgments}

Prof. Dr. A. Alzola in memoriam. We would like to thank Prof. Dr. Jacques Rieumont for support and encouragement for this research. One of the authors (JMNV) thanked the CEIICH and the Institute of Physics of the UNAM, Mexico for the warm hospitality. Finally, we thank the anonymous reviewers for their helpful comments and interesting suggestions.

\section{Conflict of interest}

The authors declare no conflict of interest.

\section{References}

1. Tam WL, Weinberg RA. The epigenetics of epithelial-mesenchymal plasticity in cancer. Nature medicine. 2013;19(11):1438.

2. Izquierdo-Kulich E, Nieto-Villar JM. Morphogenesis and complexity of the tumor patterns. in without bounds: a scientific canvas of nonlinearity and complex dynamics. Springer-Verlag Berlin Heidelberg. 2013;657691.

3. Jemal A, Siegel R., Ward E, et al. Cancer statistics. CA: a cancer journal for clinicians. 2006;56(2):106-130. 
4. Kalluri R, Weinberg RA. The basics of epithelial-mesenchymal transition. The Journal of clinical investigation. 2010; 120(5):1786-786.

5. Guerra A, Rodriguez DJ, Montero S, et al. Phase transitions in tumor growth VI: epithelial-mesenchymal transition. Physica A: Statistical Mechanics and its Applications. 2018;499:208-215.

6. Moreno-Bueno G, Portillo F, Cano A. Transcriptional regulation of cell polarity in EMT and cancer. Oncogene. 2008;27(55), 6958.

7. Salami J, Crews CM. Waste disposal-an attractive strategy for cance therapy. Science. 2017;355(6330):1163-1167.

8. Kalluri R, Weinberg RA. The basics of epithelial-mesenchymal transition. The Journal of clinical investigation. 2009;119(6), 1420-1428.

9. Yang J, Weinberg RA. Epithelial-mesenchymal transition: at the crossroads of development and tumor metastasis. Developmental cell. 2008;14(6):818-829.

10. Scheel C, Weinberg, RA. Cancer stem cells and epithelial-mesenchymal transition: concepts and molecular links. In Seminars in cancer biology. Academic Press. 2012;22(5-6):396-403.

11. Mormont MC, Levi F. Cancer chronotherapy: principles, applications and perspectives. Cancer. 2003;97(1):155-169.

12. Levi F. Circadian chronotherapy for human cancers. The lancet oncology 2001;2(5):307-315.

13. Betancourt-Mar JA, Nieto-Villar JM. Theoretical models for chronotherapy: periodic perturbations in funnel chaos type. Mathematical biosciences and engineering. 2007;4(2):177-186.

14. Li XM, Mohammad-Djafari A, Dumitru M. et al. A circadian clock transcription model for the personalization of cancer chronotherapy. Cancer research. 2013;73(24):7176-7188.

15. Silva E, Martin RR, Pomuceno JP, et al. Dose and frequency in cancer therapy. Theoretical non-autonomous model of p53 network. Biological Rhythm Research. 2019;50(3):483-489.

16. Jaime JC, Mesa-Álvarez MD, Martin RR., et al. Chronotherapy of cancer: periodic perturbations in vascular growth and metastasis. Biological Rhythm Research. 2008;1-10.

17. Strogatz SH, Stewart I. Coupled oscillators and biological synchronization Scientific American. 1993;269(6):102-109.

18. Magi S, Iwamoto K, Okada-Hatakeyama M. Current status of mathematica modeling of cancer-From the viewpoint of cancer hallmarks. Current Opinion in Systems Biology. 2017;2:39-48.

19. Araujo RP, McElwain DS. A history of the study of solid tumour growth: the contribution of mathematical modelling. Bulletin of mathematical biology. 2004;66(5):1039-1091.
20. Rosenberg SA, Yang JC, Restifo NP. Cancer immunotherapy: moving beyond current vaccines. Nature medicine. 2004; 10(9):909.

21. Rosenberg SA, Packard BS, Aebersold PM, et al. Use of tumorinfiltrating lymphocytes and interleukin-2 in the immunotherapy of patients with metastatic melanoma. New England Journal of Medicine. 1988;319(25):1676-1680.

22. Adams GP, Weiner LM. Monoclonal antibody therapy of cancer. Nature biotechnology. 2005;23(9):1147.

23. Brú A, Albertos S, Subiza JL, et al. The universal dynamics of tumor growth. Biophysical journal. 2003;85(5):2948-2961.

24. Nasir NA. Selected aspects of cancer progression: metastasis, apoptosis and immune response. Springer Science \& Business Media. 2008(11)

25. MacLean AL, Harrington HA, Stumpf MP, et al. Epithelial-mesenchymal transition in metastatic cancer cell populations affects tumor dormancy in a simple mathematical model. Biomedicines. 2014;2(4):384-402.

26. Gaspard P, Burghardt I. Chemical reactions and their control on the femtosecond time scale: 20th solvay conference on chemistry. John Wiley \& Sons. 2009(220).

27. Nieto-Villar JM, E Izquierdo-Kulich, JA Betancourt-Mar, et al. Complejidad y auto-organización de patrones naturales. Cuba; 2013.

28. Joel JP, Harishankar MK, Arumugam A, et al. Hypoxia induced EMT: a review on the mechanism of tumor progression and metastasis In OSCC. Oral Oncology. 2018;80:23-32.

29. Blando J, Sharma A, Higa MG, et al. Comparison of immune infiltrates in melanoma and pancreatic cancer highlights vista as a potential target in pancreatic cancer. PNAS. 2019;116(5):1-6.

30. Marimuthu K. Epithelial mesenchymal transition as targets for cancer therapy epithelial mesenchymal transition as targets for cancer therapy. Nature Cell Biology. 2017;3(1).

31. Kröger C. Acquisition of hybrid E/M state is essential for tumorigenicity of basal breast cancer cells. Proceedings of the National Academy of Sciences. 2019;116(15):7353-7362.

32. Zañudo JG. Towards control of celular decision-making networks in the epitelial-to-mesenchymal transition. Phys Biol. 2019;16(3):031002.

33. Roseweir AK, Kong CY, Park JH, et al. A novel tumor-based epithelialto-mesenchymal transition score that associates with prognosis and metastasis in patients with stage II/III colorectal cancer. International Journal of Cancer. 2019;144(1):150-159. 